\title{
BMJ Open Retrospective analysis of medical malpractice claims in tertiary hospitals of China: the view from patient safety
}

\author{
Heng Li (1D , ${ }^{1,2}$ Shengjie Dong (D) , ${ }^{1,2}$ Ziyi Liao, ${ }^{1}$ Yao Yao (D) , ${ }^{3}$ Suwei Yuan, ${ }^{3}$ \\ Yujie Cui, ${ }^{3}$ Guohong Li (1) 1,2,3
}

To cite: Li H, Dong S, Liao Z, et al. Retrospective analysis of medical malpractice claims in tertiary hospitals of China: the view from patient safety. BMJ Open 2020;10:e034681. doi:10.1136/ bmjopen-2019-034681

- Prepublication history for this paper is available online. To view these files, please visit the journal online (http://dx.doi. org/10.1136/bmjopen-2019034681).

HL and SD contributed equally.

Received 01 October 2019 Revised 14 August 2020 Accepted 19 August 2020

A) Check for updates

(c) Author(s) (or their employer(s)) 2020. Re-use permitted under CC BY-NC. No commercial re-use. See rights and permissions. Published by BMJ.

${ }^{1}$ School of Public Health, Shanghai Jiao Tong University School of Medicine, Shanghai, China

${ }^{2}$ Center for HTA, China Hospital Development Institute, Shanghai Jiao Tong University, Shanghai, China

${ }^{3}$ China Hospital Development Institute, Shanghai Jiao Tong University, Shanghai, China

Correspondence to

Guohong Li;

guohongli@sjtu.edu.cn

\section{ABSTRACT}

Objectives The study analysed medical malpractice claims to assess patient safety in hospitals. The information derived from malpractice claims reflects potential risks and could help lead to reducing medical errors and improving patient safety.

Design, setting We analysed 4380 medical malpractice claims from 351 grade-A tertiary hospitals in China for 2008-2017. We examined the characteristics of medical errors and patient safety, including the types of medical errors, proportionate liabilities and payments for medical malpractice in different clinical specialties.

Main outcome measures We assessed claim characteristics, payment amounts and liability. Results Our data analysis demonstrated that $72.5 \%$ of the claims involved medical errors, with average payments of US\$31430. The hospital's errors in medical malpractice resulted in $41.4 \%$ average liability in patient injury payments. Most medical malpractice cases occurred in Shanghai (817 claims, 18.7\%) and Beijing (468 claims, $10.7 \%)$. The highest risks for medical error and malpractice claims were related to orthopaedics $(11.3 \%$ of all claims, $72.8 \%$ with medical errors) and obstetrics and gynaecology $(10.0 \%$ of all claims, $76.0 \%$ with medical errors). The highest rates related to proportionate liabilities were observed in otolaryngology $(51.9 \%)$ and endocrinology (47.7\%). Respiratory medicine had the highest proportion of claims in death rates $(77.3 \%)$. Medical technology errors accounted for $91.8 \%$ of the claims and medical ethics errors for $5.8 \%$. The highest average payment was found in cardiovascular surgery (US\$41733) and the lowest in stomatology (US\$8822). Conclusions A previous study found that grade-A tertiary hospitals in China have similar medical error rates to general Chinese hospitals. ${ }^{36}$ Different specialties had different risk characteristics regarding medical errors, payments and proportionate liabilities. Orthopaedics had the highest number of malpractices claims and higher proportionate liability but lower death rates.

\section{INTRODUCTION}

WHO has stated that patient safety is a global public health issue ${ }^{12}$; it has defined patient safety as preventing medical or health professionals' errors and adverse effects that may harm patients. ${ }^{1}$ The medical profession, a profession directly related to this concept,

\section{Strengths and limitations of this study}

- This is the first case study to identify the risk of medical error, proportionate liability and payment among different medical specialties in hundreds of tertiary hospitals in China.

- The retrospective method enables us to identify the characteristics of medical malpractice claims such as the region, time distribution, types of medical error and injury outcomes.

- Our study provides a new view from patient safety of understanding medical malpractice in China.

- The risks of medical errors regarding patient safety may not have been analysed comprehensively from all perspectives.

has been characterised as a high-risk profession. ${ }^{3}$ Thus, it is necessary to devote time and resources to investigating possible malpractices and adverse events that would certainly help increase patient safety. ${ }^{4}$ Furthermore, an increasing focus on patient safety has emerged in recent research alongside growing interest in aetiology and the consequences of adverse events in healthcare. ${ }^{5}$

According to a report in the National Health Commission of the People's Republic of China's yearbook, there were 9.88 million health professionals ${ }^{6}$ and 100700 medical disputes in China in $2016^{7}$; thus, on average, per 98 health professionals face a medical dispute every year. Accordingly, medical liability ranks third with respect to tort liability in China. ${ }^{8}$ These data reflect a medical malpractice crisis in China similar to that in the USA, where physicians are frequently sued $^{9}$; however, in the USA there may be 20 times more hospital adverse events than there are malpractice claims. ${ }^{10}$

Improvements in patient safety care may help end the medical malpractice crisis in China. In 2016, about 208000 medical malpractice claims were in litigation ${ }^{11}$; they accounted for about $20 \%$ of the medical 
disputes that year. Malpractice claims may represent the tip of the iceberg for patient safety problems, but they constitute adverse medical events. In China, the lack of publicly available data is a major obstacle to assessing patient safety. Malpractice claims reveal patient safety problems from the patients' perspective. Healthcare providers lack the incentive to report fully and honestly unless they are sued. Thus, an analysis of completed lawsuits on medical malpractice claims could help identify priority areas where actions can reduce medical malpractice. ${ }^{12}$

Studies of medical liability claims have directed great attention to the following topics: how to reduce medical errors $^{13-15}$; how to avoid medical damage ${ }^{16}$; how to increase the ability to recognise medical malpractice ${ }^{17}$; how to continuously improve the quality of medical services $^{18}$ and how to achieve satisfactory levels of patient safety. ${ }^{519-21}$ On the basis of previous research, we concluded that a better understanding is needed of the relationship among adverse health outcomes, medical malpractice and patient safety. Studies have confirmed that patient safety and healthcare quality have become major health policy issues in several countries-especially correlations between patient safety and medical malpractice. ${ }^{9}$ One report from California identified a strong correlation between patient safety outcomes and the volume of medical malpractice data for 2001-2005. ${ }^{22}$ In many countries, patient safety measures also involve legal perspectives ${ }^{19}$; they include tort liability for medical malpractice, which is at the intersection between patient safety and law on medical malpractice. ${ }^{23}$ In addition, the tort law system theoretically provides physicians with appropriate incentives to reduce patient injury. ${ }^{24}$

China supports the fault principle of tort law in medical malpractice claims. Information about medical errors can be collected about such claims. The present study aimed to identify tort liabilities and causes of medical error in medical malpractice claims towards helping to determine opportunities to improve safety care in China. Hospitals are supposed to investigate patient injuries, gather information relevant to safety care from medical malpractice claims and provide that information to healthcare professionals. ${ }^{25}$ Such actions should improve patient healthcare and safety. Most research has focused on avoiding medical malpractice claims towards improving patient safety in a single medical specialty. ${ }^{26-31}$ In this study, we conducted a comprehensive analysis of the cumulative effects of medical malpractice ${ }^{4}$ and compared the results among different medical specialities. Our analysis of medical malpractice claims emphasised advancing patient safety in hospitals; information about malpractice claims can provide an early warning to healthcare professionals of possible risks, which should lead to fewer medical errors and improved patient safety in China.
MATERIALS AND METHODS

\section{Sample and data collection}

This research was a retrospective cohort study of medical malpractice claims based on secondary data analyses. In 2016, there were 2232 tertiary hospitals listed in the China health statistics yearbook. Among those, 351 grade-A tertiary hospitals were listed as top-ranking hospitals according to standards provided by public authorities, including the Hospital Management Institute of Fudan University (http:/ / www.fudanmed.com/institute/), Chinese Academy of Medical Sciences (http://news.sciencenet.cn/htmlnews/ 2014/12/310441.shtm), Peking University (http://yygl. bjmu.edu.cn/zxxx/176750.htm) and Asclepius Healthcare in Hong Kong (http://www.ailibi.com/web/rank). Those 351 hospitals serve the whole of society. They are regarded as providing better healthcare and serve as benchmarks for other hospitals.

We retrieved a list of lawsuits from a computerised legal database, Chinese Justice Legal Application Support System (CJLASS) (http:// oldwenshu.court.gov.cn/). The CJLASS is a platform established by China's Supreme People's Court for the disclosure of lawsuit information; it is widely used by Chinese law professionals. We conducted a search for lawsuits using the name of each of the 351 hospitals as a keyword on the CJLASS website. We found 4558 medical malpractice lawsuits directly related to those 351 hospitals in the CJLASS from 1 January 2008 to 31 December 2017 (figure 1).

We were able to obtain legal and medical data from 4380 of those lawsuits. Most claims were related to patient safety. Legal data included whether there was financial compensation for patients and its value. Medical data included the medical specialty and proportionate damage liability.

\section{Variables}

Classification of medical specialties

According to the rule on hospital administration in China, tertiary hospitals meet or exceed regional hospital standards; they provide high-level specialised medical and health services to several regions. Tertiary hospitals have strong teams of medical professionals; only general surgery, cardiovascular surgery, thoracic surgery and neurosurgery are independently classified. We categorised all the cases into 25 medical specialty categories based on the admission specialty according to doctors' judgements.

\section{Medical fault liability}

When a medical malpractice lawsuit is litigated in China, tort liability law rules as follows: if a patient suffers any harm during medical diagnosis and treatment or if the medical institution or any of its medical professionals are at fault, the medical institution assumes compensatory liability. ${ }^{32}$ Hence, in our analysis of medical malpractice lawsuits, data related to payment signified that there was medical fault liability, that is, a medical error occurred during diagnosis or treatment. 


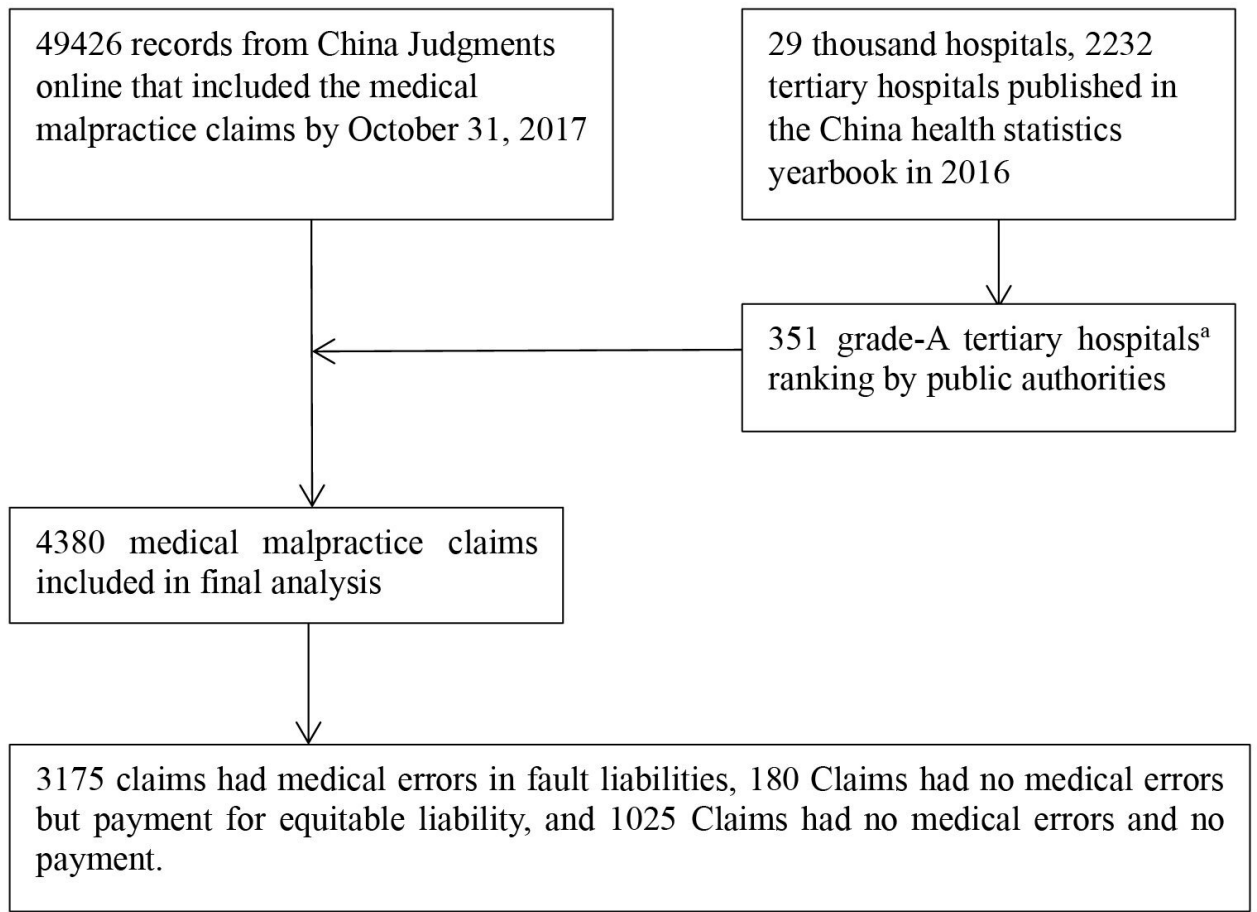

Figure 1 Flowchart of selection of medical malpractice claims in China for 2008-2017. ${ }^{\text {a The }} 351$ grade-A tertiary hospitals studied amounted to only $1.2 \%$ of Chinese hospitals; however, their number of outpatients was estimated to be 58 million in 2016; the data were gathered mainly from the hospitals' official websites. The figure of 58 million amounts to $7.3 \%$ of the nation's total outpatients and inpatients (7.93 billion, data derived from the China health statistics yearbook).

\section{Proportionate liability}

If an error related to a medical or health-related professional is found in a medical malpractice lawsuit, it does not mean that the hospital assumes full responsibility for paying the compensatory value. An expert appraisal group analyses, among other factors, the degree of liability of medical negligence related to the damage. Thus, every lawsuit receives a specific responsibility proportion (from $0 \%$ to $100 \%$ ) according to the degree of medical liability. ${ }^{33}$

In this study, following Article 36 of Interim Measures for Technical Appraisal of Medical Malpractice in China (2002), we divided proportionate liability into five categories. Full liability signified that the damage was entirely caused by medical error; thus, $100 \%$ proportionate liability was applied. Main liability meant that the damage was mainly caused by medical error, and other factors played a secondary role; accordingly $51 \%-99 \%$ proportionate liability was applied. Equal liability indicated that the damage was equally caused by medical error and other factors; therefore, $50 \%$ proportionate liability was applied. Secondary liability signified that the damage was mostly caused by other factors, and medical error played a secondary role; accordingly, $11 \%-49 \%$ proportionate liability was applied. Slight liability meant that the damage was mainly caused by other factors, and medical error played a minor role; thus, $\leq 10 \%$ proportionate liability was applied.

\section{Patient injury}

In this study, we followed the Regulation of Medical Accident Grading Standard in China (for Trial Implementation since 2002) and the Standards Assessment of Working Capacity and Grades of Employees' Disabilities Caused by Work-Related Injuries and Occupational Diseases (GB/T 16180-2014). We divided possible injuries from medical errors into four categories: death, serious injury, minor injury and mental injury only. Death is self-explanatory and requires no further clarification. A serious injury is characterised as causing any type of body disability, appearance loss, hearing loss, vision loss, organ functionality loss or other injury causing great harm to the patient's health (disability level of 1-6). A minor injury is characterised as physical or facial impairment, partial hearing loss, partial vision loss, partial organ functionality loss or other injury causing moderate damage to the patient's health (injury below disability level 6). ${ }^{18} 34$ Mental injury is characterised by psychic pain and does not include a serious or minor injury. If a patient died or suffered a serious or minor injury, mental damage may also apply; it may affect both the patient and people directly related to them (in the case of serious or minor injuries) or only to people directly related to the patient (in the case of death).

\section{Data analysis}

The descriptive analysis included frequency distribution, histogram and bubble diagram analysis. We analysed data using the PASW statistics software package, V.18.0. To account for inflation, we adjusted all renminbi amounts to the value in 2017 using the Consumer Price Index. The amounts of financial compensation presented are estimated based on the conversion rate of US $\$ 1=\mathrm{RMB} 6.51$. 
Table 1 Distribution of medical malpractice in grade-A tertiary hospitals in China $(\mathrm{N}=4380)$

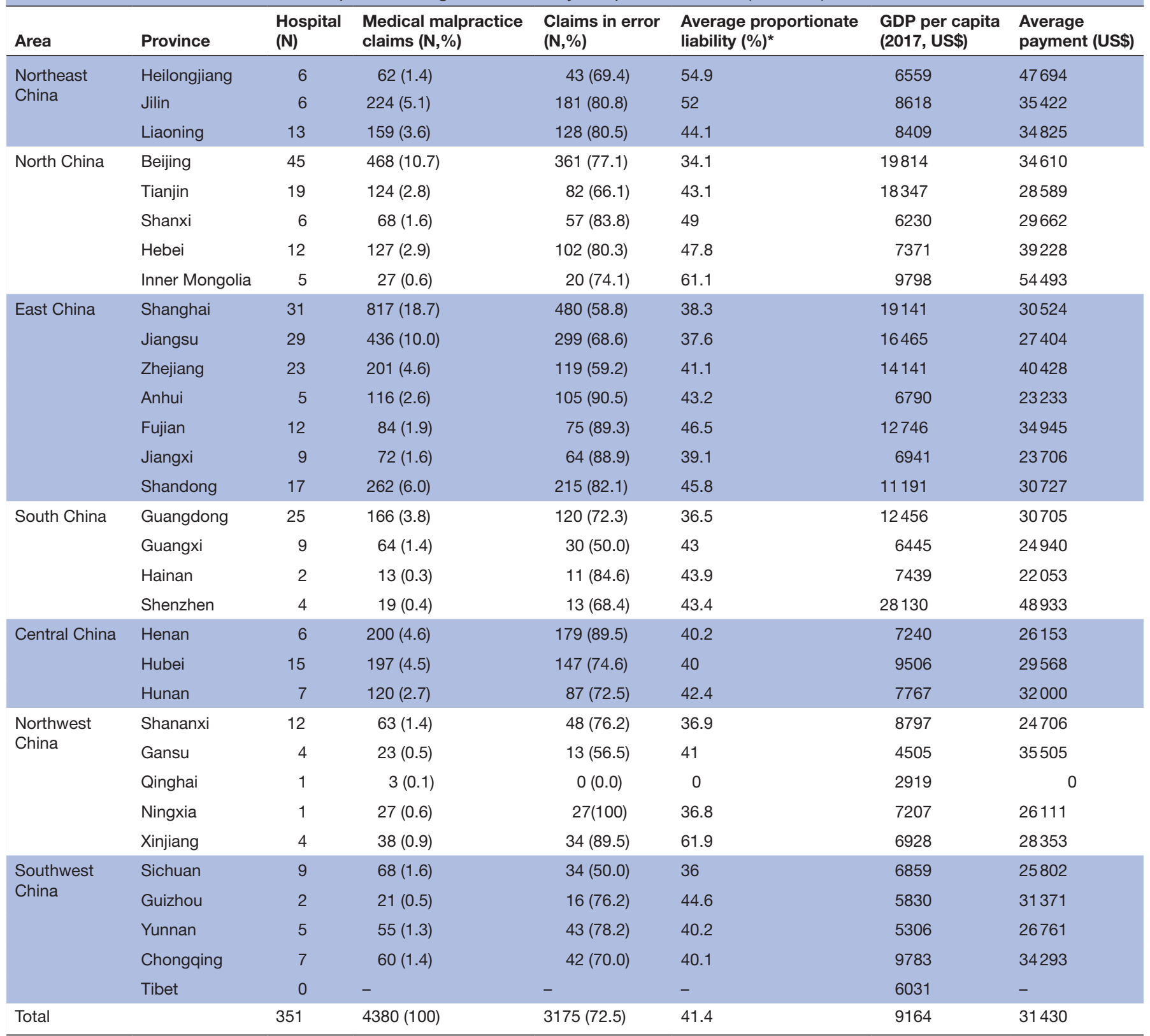

*We analysed average proportionate liability and average GDP data using the Spearman correlation test in the 14 provinces with over 100 medical malpractice claims; Spearman correlation coefficient $r=-0.65, p=0.022$.

GDP, Gross Domestic Product.

\section{Patient and public involvement}

Patients or the public were not involved in the design, or conduct, or reporting, or dissemination of our research.

\section{RESULTS}

\section{Area distribution of medical malpractices in grade-A tertiary} hospitals of China

Among the 4380 claims analysed, $72.5 \%$ (3175 claims) had medical errors in terms of fault liability (table 1); $4.1 \%$ (180 claims) had no medical errors but payments for equitable liability and 23.4\% (1025 claims) had no medical errors and no payments. The highest rates of medical malpractice claims were as follows: Shanghai (31 hospitals, 817 claims);
Beijing (47 hospitals, 468 claims) and Jiangsu province (29 hospitals, 436 claims). Proportionally, the highest rates of medical errors were found in Ningxia (100\%), Anhui $(90.5 \%)$ and Henan provinces $(89.5 \%)$; the lowest rates of medical errors were in Guangxi (50.0\%), Sichuan (50.0\%) and Gansu provinces $(56.5 \%)$.

Though $72.5 \%$ of the claims involved medical errors, the proportionate liability for medical error for the damage was not very high; the average was $41.4 \%$. The lowest rates for proportionate liability were in Beijing $(34.1 \%)$, Sichuan $(36.0 \%)$ and Guangdong (36.5\%). The highest rates for proportionate liability were in Xinjiang $(61.9 \%)$ and Mongolia (61.1\%); the provinces with high 

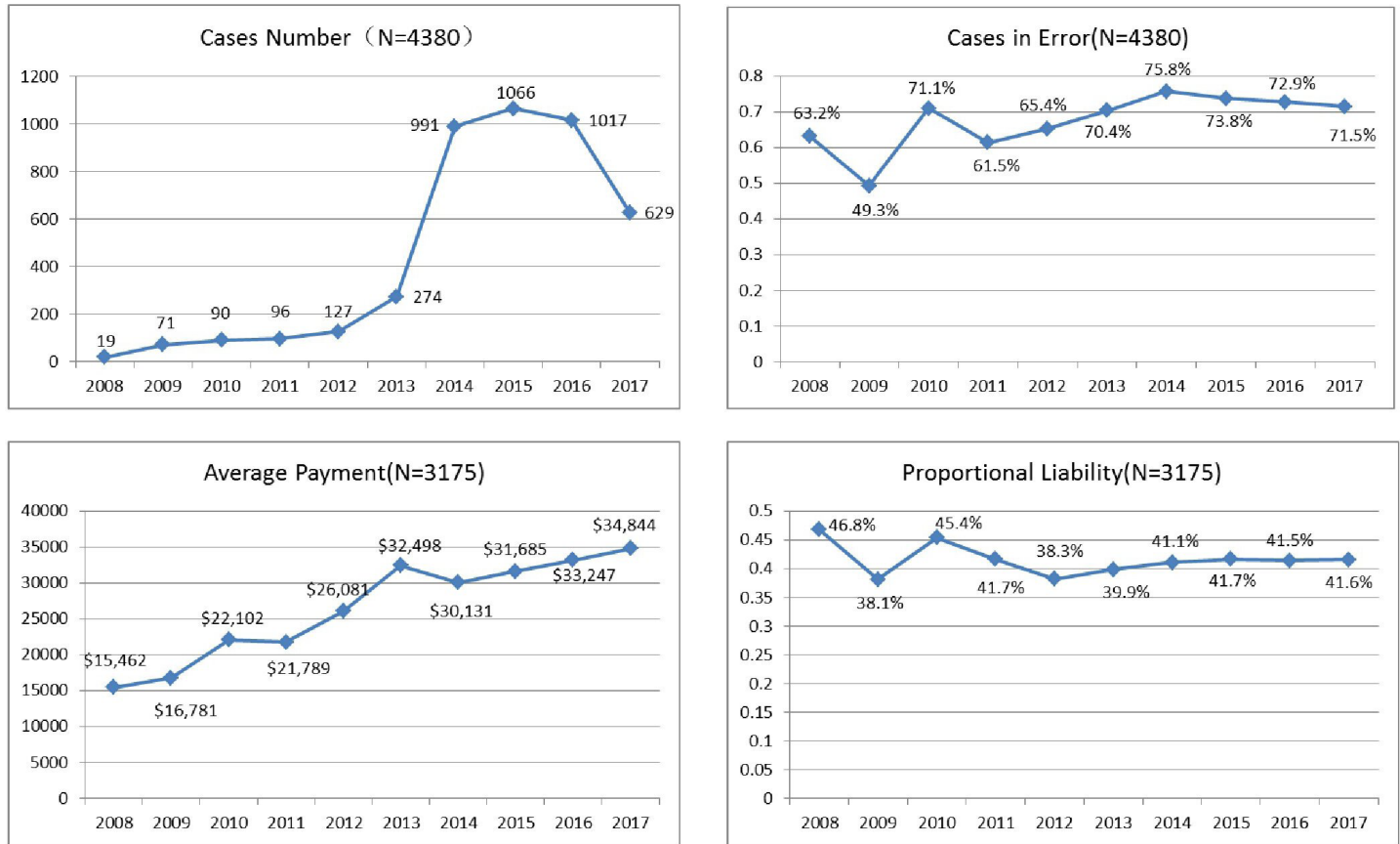

Figure 2 Time trend of medical malpractices in China's grade-A tertiary hospitals for 2008-2017.

average payments were Inner Mongolia (US\$54493), Shenzhen (US\$48933) and Heilongjiang (US\$47694).

\section{Time trend of medical malpractices in grade-A tertiary hospitals of China from 2008 to 2017}

According to time-trend analysis, the number of medical malpractices increased greatly from 2014: almost 1000 claims annually were evident among all the grade-A Chinese tertiary hospitals; the maximum was 1065 in 2015 (figure 2). The proportion of medical error claims ranged from $49.3 \%$ in 2009 to $75.8 \%$ in 2014 . The proportionate liability did not vary greatly from 2008 to 2017 : the range was $38.1 \%-46.8 \%$. However, payments for malpractice claims rose continuously from 2008 to 2017: the maximum annual average payment was US\$34844 in 2017; that was over twice the average payment registered in 2008 (US\$15 462).

\section{Medical malpractice claims in different specialties}

We found that the medical specialties where medical errors were more likely were as follows: orthopaedics (497 claims, 11.3\%); obstetrics and gynaecology (437 claims, $10.0 \%$ ) and emergency medicine (422 claims, 9.6\%) (figure 3). The specialties that accounted for most claims were general surgery $(8.0 \%)$, gastroenterology $(8.0 \%)$, oncology $(6.8 \%)$ and cardiovascular surgery $(5.3 \%)$; they accounted for $59.1 \%$ of all claims (2589 of 4380 claims). The specialties with the highest medical error risks were thoracic surgery $(85.9 \%)$, oncology $(78.5 \%)$, gastroenterology $(77.8 \%)$ and obstetrics and gynaecology (76.0\%); the average medical error risk was $72.5 \%$ among all specialties. The specialties with the lowest medical errors were stomatology (54.8\%), haematology (54.8\%) and psychiatry (56.4\%). Among all clinical specialties, the range was $54.8 \%-85.9 \%$.

\section{Patient injury risk in different specialties}

In terms of patient injury, 3175 claims involved damage caused by medical errors. Death was the outcome in 1452 claims $(45.7 \%)$, serious injury in 879 claims $(27.7 \%)$, minor injury in 790 claims (24.9\%) and mental damage in 54 claims $(1.7 \%)$ (table 2). Regarding patient injury risks in different medical specialties, death had the highest proportional outcome in psychiatry $(84.2 \%)$, respiratory medicine $(77.3 \%)$ and haematology $(70.6 \%)$; death had the lowest proportional outcome in plastic surgery $(5.0 \%)$, stomatology $(6.9 \%)$, ophthalmology $(10.0 \%)$ and orthopaedics $(16.9 \%)$. We also found that the mortality rates were more related to the patients' original disease status rather than medical errors (figure 4). Plastic surgery showed the highest proportion $(70.0 \%)$ among medical specialties in terms of minor injury. When intrauterine foetal death occurred in pregnancy but the mother was unharmed, there was often only mental damage $(6.9 \%$, that is, 23 of all 332 cases in obstetrics and gynaecology).

\section{Proportionate liability and payment in different specialties}

In terms of proportionate liability of hospital staff errors in medical malpractice claims, the average was $41.4 \%$. Furthermore, 400 medical error claims $(12.6 \%)$ of all 3175 medical error claims had slight proportionate liabilities ( $\leq 10 \%$ proportionate liability); 1531 medical error claims $(48.2 \%)$ had minor proportionate liabilities (11\%-49\% proportionate liability); 330 claims (10.4\%) had equal liabilities (50\% proportionate liability); 810 claims $(25.5 \%)$ had main liabilities $(>50 \%$ proportionate liability) and 104 claims (3.3\%) had full liabilities $(100 \%$ proportionate liability) caused by medical errors.

Regarding specialties, psychiatry had the lowest proportionate liability (29.4\%); the average payment there 


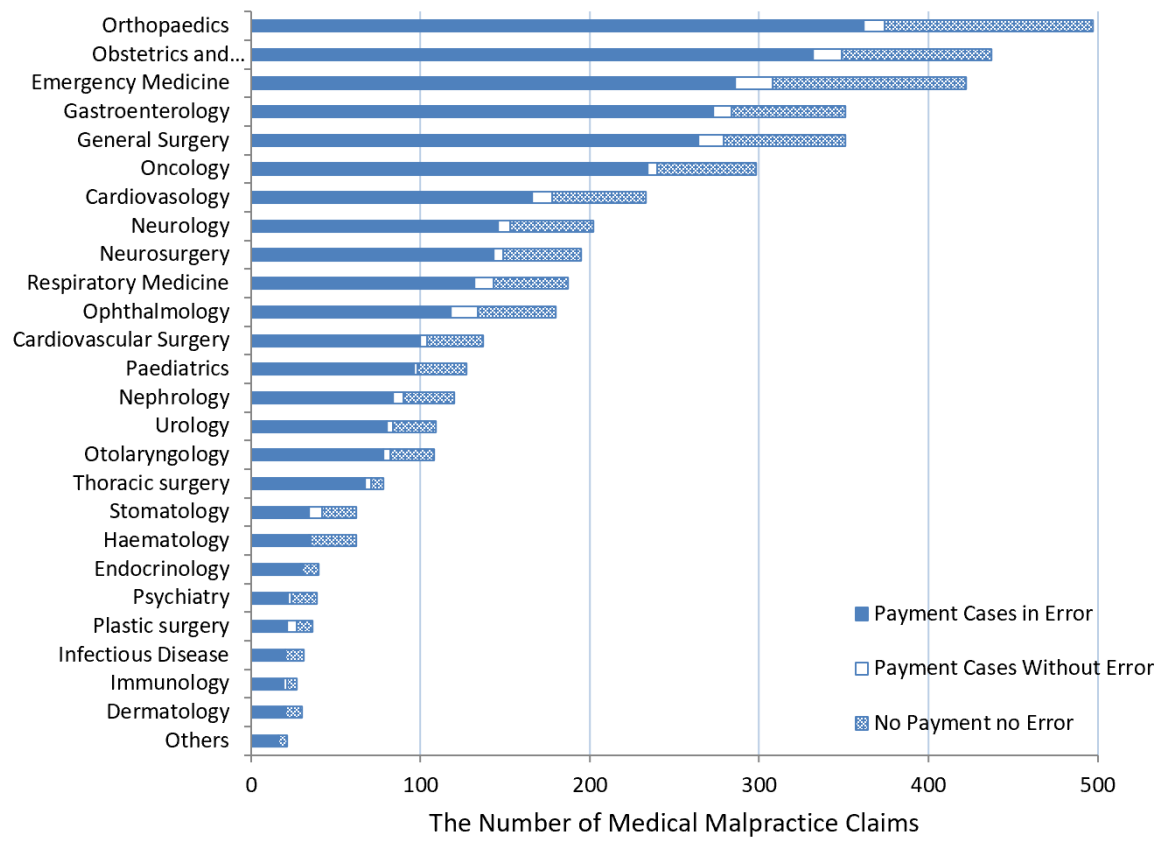

Figure 3 Medical error risk outcomes in different clinical specialties $(N=4380)$.

was $\$ 25176$ (figure 5). In terms of average payment for medical error among the specialties, stomatology had the lowest (US\$8820) and cardiovascular surgery the highest average payment (US\$41 733).

With respect to proportionate liability within the specialties in medical malpractice claims, the highest average was found in otolaryngology ( $51.9 \%$ proportionate liability). The specialties with higher proportionate liability in medical malpractice claims were endocrinology (average $47.9 \%$ proportionate liability), orthopaedics (average $46.5 \%$ ), general surgery (average $44.7 \%$ ), obstetrics and gynaecology (average 43.9\%) and gastroenterology (average 43.6) (figure 5). For all the specialties, the average proportionate liability ranged from $29.4 \%$ to $51.9 \%$; the average payment was US $\$ 8820-U S \$ 41733$.

\section{Types of medical error in malpractice claims}

The distribution of injuries and associated proportionate liability by type of error appears in table 2. Of all 3175 medical error claims, $2914(91.8 \%)$ were due to a medical technology error. In all, 183 claims $(5.8 \%)$ were medical ethics errors; $28(0.9 \%)$ were medical product errors and $50(1.6 \%)$ were medical management errors. In terms of reason for payment, the most common type was errors related to treatment $(36.1 \%)$, followed by errors related to surgery $(30.1 \%)$ and mistakes related to diagnosis $(13.7 \%)$. The mean proportionate liability in different medical errors ranged from $6.2 \%$ to $61.5 \%$. The mean proportionate liability related to payments attributed to medical errors was the highest rate with product errors $(52.5 \%)$; medical technical errors were the lowest $(40.7 \%)$.

\section{DISCUSSION}

Medical malpractice area and time trend in grade-A tertiary hospitals of China

We found that provinces with higher Gross Domestic Product (Beijing, Tianjin, Shanghai and Chongqing) had the lowest proportionate liability in medical malpractice claims (under 30\%). Thus, it may be inferred that there is better patient safety and healthcare quality in more economically developed areas.

Previous studies have determined that not all adverse health outcomes can be attributed to medical errors. ${ }^{18}$ Our finding that the average proportionate liability caused by medical errors was $34.52 \%$ is in agreement with such study results. From our results about malpractice claims for 2008-2017, other than increased payments for malpractice claims, we observed no changes in medical error rates and proportionate liability in malpractice claims. The proportionate liability in such claims could reflect patient safety. Thus, from our analysis of proportionate liability and evidence-based malpractice claims, we may conclude that patient safety in grade-A tertiary hospitals did not improve greatly over the study period: the proportionate liability was about $40 \%$ for 2008-2017. It should be noted that a previous study found that China had gradually improving healthcare access and quality from 2005 to $2015 .^{35}$ Therefore, grade-A tertiary hospitals should focus more on medical malpractice and errors if they aim to improve patient safety and healthcare quality in China and in their own organisations.

Medical law reform was introduced (tort law) in 2010. The original goal of the tort law was to create alignment between decreased malpractice claims and improved patient safety. Our findings concur with those of Swift, 
Table 2 Injury outcomes and association with proportionate liability by type of error $(\mathrm{N}=3175)$

\begin{tabular}{|c|c|c|c|c|c|c|}
\hline \multirow[b]{2}{*}{ Type of error } & \multicolumn{4}{|c|}{ Injury outcome } & \multirow[b]{2}{*}{ Total $(\mathrm{N}, \%)$} & \multirow[b]{2}{*}{$\begin{array}{l}\text { Proportionate } \\
\text { liability (\%) }\end{array}$} \\
\hline & Minor injury & $\begin{array}{l}\text { Severe } \\
\text { injury }\end{array}$ & Death & $\begin{array}{l}\text { Emotional } \\
\text { injury only }\end{array}$ & & \\
\hline Medical technology error & 719 & 823 & 1332 & 40 & 2914 (91.8) & 42.4 \\
\hline Diagnosis related & 100 & 112 & 213 & 10 & $435(13.7)$ & 39.4 \\
\hline Treatment related & 283 & 276 & 578 & 9 & $1146(36.1)$ & 41.5 \\
\hline Drugs use related & 15 & 19 & 50 & 1 & $85(2.7)$ & 36.6 \\
\hline Surgery related & 262 & 331 & 353 & 10 & $956(30.1)$ & 46.3 \\
\hline Nursing related & 1 & 8 & 25 & 0 & $34(1.1)$ & 38 \\
\hline Infection related & 11 & 3 & 30 & 0 & $44(1.4)$ & 39.6 \\
\hline Pregnancy and delivery related & 37 & 58 & 49 & 10 & $154(4.9)$ & 43.4 \\
\hline Anaesthesia related & 2 & 0 & 0 & 0 & $2(0.1)$ & 25 \\
\hline Monitor related & 3 & 5 & 14 & 0 & $22(0.7)$ & 34.3 \\
\hline Other & 5 & 11 & 20 & 0 & $36(1.1)$ & 27.4 \\
\hline Medical ethics error & 47 & 48 & 74 & 14 & $183(5.8)$ & 27.4 \\
\hline Informant & 44 & 44 & 70 & 13 & $171(5.4)$ & 26.2 \\
\hline Privacy & 2 & 0 & 0 & 0 & $2(0.1)$ & 79.9 \\
\hline Other & 1 & 4 & 4 & 1 & $10(0.3)$ & 38.8 \\
\hline Medical product error & 17 & 5 & 6 & 0 & $28(0.9)$ & 54.4 \\
\hline Blood and blood products & 10 & 4 & 0 & 0 & $14(0.4)$ & 65 \\
\hline Drugs & 2 & 0 & 6 & 0 & $8(0.3)$ & 30.5 \\
\hline Medical equipment & 5 & 1 & 0 & 0 & $6(0.2)$ & 61.5 \\
\hline Medical management error & 7 & 3 & 40 & 0 & $50(1.6)$ & 22.1 \\
\hline Administrative management & 4 & 1 & 16 & 0 & $21(0.7)$ & 24.4 \\
\hline Medical record management & 1 & 1 & 1 & 0 & $3(0.1)$ & 50 \\
\hline Risk management & 1 & 0 & 16 & 0 & $17(0.5)$ & 19.6 \\
\hline Other & 1 & 1 & 7 & 0 & $9(0.3)$ & 12.4 \\
\hline Total & $790(24.9)$ & 879 (27.7) & $1452(45.7)$ & $54(1.7)$ & 3175 (100) & 41.4 \\
\hline
\end{tabular}

who found little evidence that the tort system improved patient safety ${ }^{17}$ in the USA.

\section{Consistent medical error risks in grade-A tertiary hospitals in China}

We found that about $76.62 \%$ of medical malpractice claims were related to medical errors; this finding is consistent with that of previous research, which analysed data for China for 1998-2011. ${ }^{36}$ However, our figure is much higher than that of other countries or regions previously examined. According to Rogers et al, only $58 \%$ of malpractice claims in the USA were through medical errors and patient injuries. ${ }^{12}$ Furthermore, one study analysed data from the US states of New York, Utah,and Colorado; it found that only $17 \%-22 \%$ of malpractice claims involved medical errors. ${ }^{10}$ Hwang et al found that in Taiwan, only $14.1 \%$ of verdicts in medical malpractice claims identified medical errors. ${ }^{16}$ In Poland, a commission analysed adverse medical events and found that $23.7 \%$ of claims were due to medical errors. ${ }^{19}$
Higher mortality but lower proportionate liability in grade-A tertiary hospitals of China

In China, tertiary hospitals receive and treat mainly patients with serious diseases. Accordingly, previous research has found patient injury resulting from medical care to be common in such hospitals; it may often be life-threatening or fatal. ${ }^{5}$ Comparing our study's results with those of Rogers $e t a l$ s, we found that serious injuries were lower $(27.7 \%)$ but the death rate $(45.7 \%)$ was higher; in their study, serious injuries amounted to $65 \%$, and the death rate was $23 \% .{ }^{12}$ One study analysed data from all types of Chinese hospitals and reported a death rate of $34.3 \% ;^{36}$ among our grade-A tertiary hospitals, the death rate was higher $(45.7 \%)$. The same phenomenon was evident with another study in Taiwan, in malpractice claims, the death rate was $36.9 \% .^{16}$ According to the National Health Service and Quality Safety Report of China, patient mortality in tertiary hospitals in 2015 $(0.71 \%)$ was also higher than in secondary hospitals $(0.48 \%) .{ }^{37}$ Thus, we conclude that among other factors, 


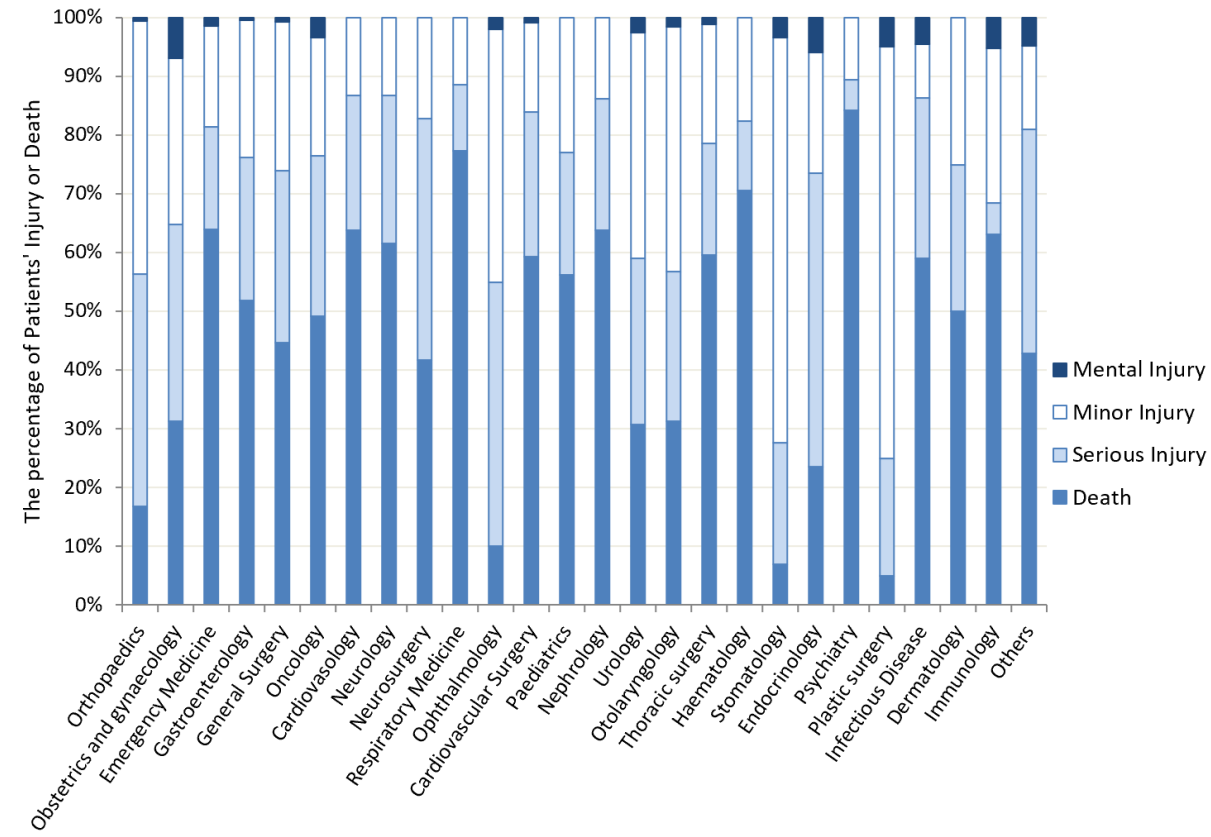

Figure 4 Outcomes of patient injuries related to medical malpractice claims in different clinical specialties $(N=3175)$.

patients' original disease status could be the main reason for adverse health outcomes.

We found that in the grade-A tertiary hospital malpractice lawsuits, $76.6 \%$ of medical error claims were due to slight, minor or equal liabilities. Renkema et al identified complexity of care as only one of four factors that could affect the relationship between medical error risks and physicians' behaviour that could reduce patient safety. ${ }^{38}$ Thus, one might infer that medical errors tend to be more frequent in complex hospital care, which is frequently the case with grade-A tertiary hospitals. That inference might be more acceptable to the general public and in court cases.

\section{Medical error risks in different specialties}

We found that the highest medical error risks in malpractice claims were in orthopaedics ( $11.3 \%$ of all claims, $72.8 \%$ of medical errors), obstetrics and gynaecology (10.0\% of all claims, $76.0 \%$ of medical errors) and emergency medicine $(9.6 \%$ of all claims, $67.8 \%$ of medical errors). Similarly, in studies conducted in Shanghai, obstetrics, general surgery and emergency medicine had

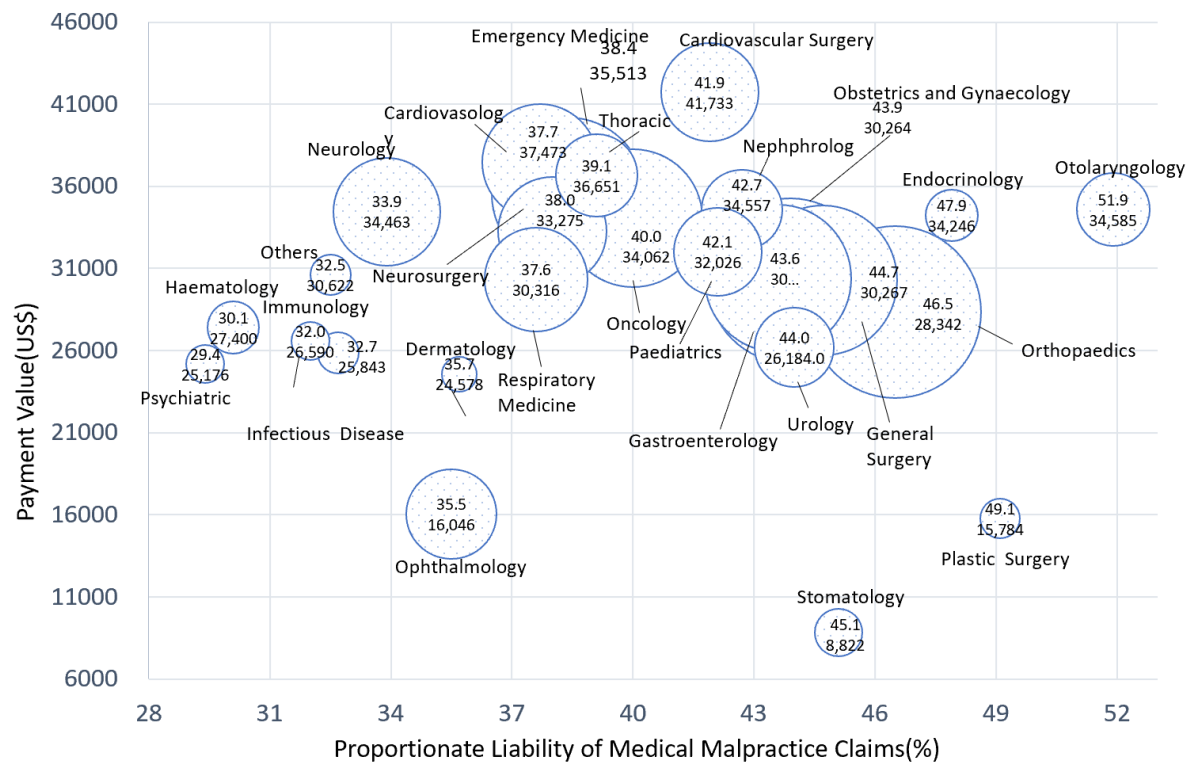

Figure 5 Proportionate liability rates and payment values of medical malpractice claims in different clinical specialties $(\mathrm{N}=3175)$. 
the highest medical error risks. ${ }^{39}{ }^{40}$ The same medical error risks were observed in the USA: the highest risk specialties commonly accounted for the worst effects of malpractice. ${ }^{9}$

One previous study of ours investigated the types of medical errors among 1086 lawsuits in China in 2014; it found that $79 \%$ of all cases with payments were attributed to medical technical errors, $7 \%$ were due to medical product errors and $7 \%$ to medical management errors. ${ }^{36}$ In the present study, the proportion of medical technical errors $(91.8 \%)$ was much higher in the grade-A tertiary hospitals; however, medical product and medical management errors $(2.5 \%$ for both) were lower than in our previous study, which was conducted in general hospitals (14\%).

One study found that about half of medical technical errors could lead to severe harm. ${ }^{41}$ Diagnosis, treatment and surgery errors were reportedly the leading types of medical errors among malpractice claims in the USA. ${ }^{42}$ Surgical mishaps are an important cause of morbidity and mortality: in developed countries, it was observed that $3 \% \%-16 \%$ of patients undergoing major surgery suffered serious complications, half of which could have been prevented. ${ }^{44}$ Thus, from the above findings, we conclude that medical malpractice poses an important threat to healthcare quality and safety in China.

In this study, we observed marked differences among the types of error in the degree of proportionate liability: there was higher proportionate liability for medical product errors (52.5\%), such as drug, transfusion and medical equipment errors in the malpractice claims. One investigation reported that $90.8 \%$ of claims could be attributed to blood and blood product errors; they resulted in severe infections. ${ }^{45}$ In the present study, the proportionate liability for such errors was the highest $(60.7 \%)$ among all types. Owing to the higher proportionate liability with medical product error claims, medical professionals should pay greater attention to such matters towards improving the safety of drugs, transfusions and medical equipment.

\section{Medical error reporting system for improving patient safety in China}

Early in 1999, a report by the Institute of Medicine, 'To Err Is Human: Building a Safer Health System', prompted growing concern about patient safety in the USA and other countries. In 2005, the Patient Safety and Quality Improvement Act (Public Law) was introduced in the USA: the act's goal was to encourage voluntary and confidential medical error reporting to improve patient safety. ${ }^{46}$ In one study, it was shown that the law was a key facilitator for integrating patient safety and risk management units in Italian healthcare facilities. ${ }^{47}$

In 2009, the Tort Law of China was introduced to address liability in medical malpractice. From a policy perspective, malpractice liability laws allow hospitals and medical staff to engage in peer-review risk management activities. ${ }^{22}$ Since 2012 , the national clinical improvement system of China has operated a reporting system for medical safety, and all national hospitals have voluntarily joined. Over 20000 events were reported through that system in $2015^{37}$; however, the results were not disclosed to the public.

Furthermore, in 2016, the Regulation of Management for Healthcare Quality was enacted by the National Health and Family Planning Commission of the People's Republic of China. In the section concerning healthcare safety risk, the regulation states that a reporting system should actively encourage medical institutions and staff to report adverse events; that would promote information sharing and ongoing improvement to prevent patient injury. ${ }^{48}$ Thus, improving safety care can help reduce malpractice claims, ${ }^{22}$ and laws can offer appropriate incentives to improve patient safety.

\section{Patient-centred hospital culture}

The present study has shown that China has experienced a surge in medical malpractice claims, arousing widespread concern among policymakers, medical professionals and the public. More seriously, with frequent reports of disgruntled patients wreaking violence on innocent doctors, workplace violence in healthcare has become a familiar occurrence. ${ }^{49} 50$ It is not clear whether the increasing frequency of such claims reflects a decrease in the general quality of care ${ }^{18}$ However, most of the claims we investigated were associated with medical technology errors. A medical technical error relates to negligence that does not conform with existing medical professional knowledge or technical levels; it is particularly indicative of a low-quality medical service.

A high risk of litigation is likely to induce defensive medical practices among doctors, which could further reduce care quality. In this study, over $90 \%$ of the cases involving death or serious injury had technology errors, which could be viewed as having a substantial negative impact on patient safety. There is a connection between a positive hospital culture and beneficial performance, and the specific management of organisational culture is possible. ${ }^{51}$ The notion of 'culture' frames healthcare institutions in 'cultural' terms, which offer useful insights towards improving healthcare processes and outcomes. Medical institutions should integrate the patient-centred concept into each process, continuously improve their medical quality, aim to avoid technical errors and ultimately ensure patient safety. ${ }^{51} 52$

\section{STUDY LIMITATIONS}

This study has several limitations. First, many adverse medical events may have been excluded from legal databases; many malpractice claims could have been settled or withdrawn before an injured patient filed a malpractice claim in court. ${ }^{53}$ The data analysed in this study were based on completed lawsuits; therefore, ongoing claims were not included in the database owing to incomplete information. Second, the reviewed medical malpractice lawsuits may not have been complete: a medical error 
record taken directly from a hospital could have affected the accuracy of the analysis. Third, the patients' detailed information was not documented in the lawsuits; thus, it is possible that the medical error risks regarding patient safety were not analysed from all perspectives. Hence, safety care and improvement of quality based on inferences from the lawsuits may not have been reflected in our research with respect to proportionate liability and medical errors.

Acknowledgements We thank Liwen Bianji, Edanz Editing China (www. liwenbianji.cn/ac), for editing the English text of a draft of this manuscript.

Contributors $\mathrm{HL}$ and GL were responsible for design of the study. The paper was primarily written by HL. Data collection and analysis were initially carried out by SD and subsequently completed by HL and ZL. SD, YY, SY, YC and GL commented on successive drafts of the paper and suggested areas for revision and improvement. $\mathrm{HL}$ and SD contributed equally to this article. All authors have read and approved the final manuscript.

Funding This study was supported by the Key Projects of Philosophy and Social Sciences Research of Ministry of Education of China (Grant No. 18JZD044), National Natural Science Foundation of China (Grant No. 71804111).

Competing interests None declared.

Patient and public involvement Patients and/or the public were not involved in the design, or conduct, or reporting, or dissemination plans of this research.

Patient consent for publication Not required.

Ethics approval This study did not involve any human participants. The data used in our analysis are publicly available. Hence, this study was not considered to require assessment by the ethics review boards of the relevant hospitals.

Provenance and peer review Not commissioned; externally peer reviewed.

Data availability statement Data are available upon reasonable request. Deidentified data that underlie the results reported in this article (text, tables, figures and supplementary material) can be available to researcher who apply for proposed use after approval by the corresponding author of this study. A signed data access agreement is also needed for data requestors.

Open access This is an open access article distributed in accordance with the Creative Commons Attribution Non Commercial (CC BY-NC 4.0) license, which permits others to distribute, remix, adapt, build upon this work non-commercially, and license their derivative works on different terms, provided the original work is properly cited, appropriate credit is given, any changes made indicated, and the use is non-commercial. See: http://creativecommons.org/licenses/by-nc/4.0/.

\section{ORCID iDs}

Heng Li http://orcid.org/0000-0003-4854-8537

Shengjie Dong http://orcid.org/0000-0002-4573-8792

Yao Yao http://orcid.org/0000-0002-1191-9843

Guohong Li http://orcid.org/0000-0003-0445-5363

\section{REFERENCES}

1 World Health Organization. WHO patient safety. Available: https:// www.who.int/patientsafety/en/ [Accessed 13 Aug 2019].

2 World Health Organization. WHO world alliance for patient safety. Available: https://www.who.int/patientsafety/worldalliance/en/ [Accessed 13 Aug 2019].

3 Ronggui C. Medical quality and patient safety in China (Chinese). Chinese Hospitals 2007;11:1-4.

4 Vincent C, Amalberti R. Safer healthcare: strategies for the real world. London: Springer International Publishing, 2016.

5 Mileder LP. Medical error and patient safety in the spotlight. Wien Klin Wochenschr 2017;129:852-3.

6 China's. Health and family planning statistics yearbook 2017. Beijing: China Union Medical University Press, 2017.

7 Zijun L. Improve doctor-patient relationship (Chinese), 2017. Available: https://www.cn-healthcare.com/article/20170308/ content-490354.html?appfrom=jkj\&from=timeline\&isappinstalled=0 [Accessed 13 Aug 2019].
8 China Judgments Online [database online]. MD: the supreme people's court of China, 2014. Available: http://wenshu.court.gov.cn/

9 Dalton GD, Samaropoulos XF, Dalton AC. Improvements in the safety of patient care can help end the medical malpractice crisis in the United States. Health Policy 2008;86:153-62.

10 Mello MM, Hemenway D. Medical malpractice as an epidemiological problem. Soc Sci Med 2004;59:39-46.

11 The Supreme People's Court of China. The number of medical disputes in China in 2016 dropped by 6.7 compared with 2015, 2017 (Chinese). Available: http://finance.sina.com.cn/roll/2017-02-23/docifyavwcv8643027.shtml [Accessed 13 Aug 2019].

12 Rogers SO, Gawande AA, Kwaan M, et al. Analysis of surgical errors in closed malpractice claims at 4 liability insurers. Surgery 2006;140:25-33.

13 Giraldo P, Sato L, Martínez-Sánchez JM, et al. Eleven-year descriptive analysis of closed Court verdicts on medical errors in Spain and Massachusetts. BMJ Open 2016;6:e11644.

14 Wu K-H, Cheng H-H, Cheng F-J, et al. An analysis of closed medical litigations against the obstetrics departments in Taiwan from 2003 to 2012. Int J Qual Health Care 2016;28:47-52.

15 Goergen S, Schultz T, Deakin A, et al. Investigating errors in medical imaging: lessons for practice from medicolegal closed claims. J Am Coll Radiol 2015;12:988-97.

16 Hwang C-Y, Wu C-H, Cheng F-C, et al. A 12-year analysis of closed medical malpractice claims of the Taiwan civil court: a retrospective study. Medicine 2018;97:e237.

17 Swift JQ. Patient safety and the malpractice system. Oral Maxillofac Surg Clin North Am 2017;29:223-7.

18 Wang Z, Li N, Jiang M, et al. Records of medical malpractice litigation: a potential indicator of health-care quality in China. Bull World Health Organ 2017;95:430-6.

19 Budzowska J, Mikos M, Juszczyk G, et al. [Patient safety in poland according to analysis of medical adverse events]. Wiad Lek 2017;70:1096-101.

20 Manuel BM, Greenwald LM. Using medical malpractice closed claims data to reduce surgical risk and improve patient safety. Bull Am Coll Surg 2007;92:27-30.

21 Pawlson LG, O'Kane ME, prevention M. Malpractice prevention, patient safety, and quality of care: a critical linkage. Am J Manag Care 2004;10:281-4.

22 Greenberg MD, Haviland AM, Ashwood JS, et al. Is better patient safety associated with less malpractice activity?: evidence from California. Rand Health Q 2011;1:1.

23 Tingle J, Bark P. Patient safety, law policy and practice. London: Routledge, 2011.

24 Schwartz WB, Komesar NK, Doctors KNK. Doctors, damages and deterrence. An economic view of medical malpractice. $N$ Engl J Med 1978;298:1282-9.

25 Sage WM. Putting the patient in patient safety: linking patient complaints and malpractice risk. JAMA 2002;287:3003-5.

26 Myers LC, Skillings J, Heard L, et al. Medical malpractice involving pulmonary/critical care physicians. Chest 2019;156:907-14.

27 Regev GS, Ser AM. Breast cancer medical malpractice litigation in New York: The past 10 years. Breast 2019;46:1-3.

28 Fogel AL, Kvedar JC. Reported cases of medical malpractice in direct-to-consumer telemedicine. JAMA 2019;321:1309-10.

29 Samuel LT, Sultan AA, Rabin JM, et al. Medical malpractice litigation following primary total joint arthroplasty: a comprehensive, nationwide analysis of the past decade. J Arthroplasty 2019;34:S102-7.

30 Glick TH, Cranberg LD, Hanscom RB, et al. Neurologic patient safety: an in-depth study of malpractice claims. Neurology 2005;65:1284-6

31 Zhou L, Li H, Li C, et al. Risk management and provider liabilities in infantile cerebral palsy based on malpractice litigation cases. $J$ Forensic Leg Med 2019;61:82-8.

32 China DOTP. Article 54 tort law of the people's Republic of China, 2010.

33 China $\mathrm{MOH}$. Article 36, rule on medical malpractice identification, 2002.

34 Supreme People's Court C. Article 3, injury degree identification standard of China, 2014

35 GBD 2015 Healthcare Access and Quality Collaborators. Electronic address: cjlm@uw.edu, GBD 2015 Healthcare Access and Quality Collaborators. Healthcare access and quality index based on mortality from causes amenable to personal health care in 195 countries and territories, 1990-2015: a novel analysis from the global burden of disease study 2015. Lancet 2017;390:231-66.

$36 \mathrm{Li} \mathrm{H}, \mathrm{Wu}$ X, Sun T, et al. Claims, liabilities, injures and compensation payments of medical malpractice litigation cases in China from 1998 to 2011. BMC Health Serv Res 2014;14:390. 
37 Commission NHAF. National health service and quality safety report of China, 2016: 46.

38 Renkema E, Broekhuis M, Ahaus K. Conditions that influence the impact of malpractice litigation risk on physicians' behavior regarding patient safety. BMC Health Serv Res 2014;14:38.

39 Meihong S, Hua Z, Qin C. Analysis on the causes, distribution and compensation of 5012 medical damage disputes (Chinese). Medical and law 2015;6:42-8.

40 Gao P, Li X, Zhao Z, et al. Diagnostic errors in fatal medical malpractice cases in Shanghai, China: 1990-2015. Diagn Pathol 2019;14:8.

41 Singh H, Meyer AND, Thomas EJ, et al. The frequency of diagnostic errors in outpatient care: estimations from three large observational studies involving US adult populations. BMJ Qual Saf 2014;23:727-31.

42 Gupta A, Snyder A, Kachalia A, et al. Malpractice claims related to diagnostic errors in the hospital. BMJ Qual Saf 2018;27:53-60.

43 Schaffer AC, Jena AB, Seabury SA, et al. Rates and characteristics of paid malpractice claims among US physicians by specialty, 19922014. JAMA Intern Med 2017;177:710-8.

44 WHO's patient-safety checklist for surgery. Lancet 2008;372:1.
$45 \mathrm{Li} \mathrm{H}$, Zhao X, Li W, et al. Claims and compensation for complications resulting from blood transfusions in China from 1998 to 2013. Transfus Apher Sci 2015;53:329-36.

46 Kinnaman K. Patient safety and quality improvement act of 2005. Orthop Nurs 2007;26:14-18.

47 Di Luca A, Vetrugno G, Pascali VL, et al. Perspectives on patient safety and medical malpractice: a comparison of medical and legal systems in Italy and the United States. J Patient Saf 2018;15:e78-81.

48 Commission NHAF. Chapter 5 regulation of management for healthcare quality of China, 2016.

49 Zhao S, Liu H, Ma H, et al. Coping with workplace violence in healthcare settings: social support and strategies. Int J Environ Res Public Health 2015;12:14429-44.

50 Jiao M, Ning N, Li Y, et al. Workplace violence against nurses in Chinese hospitals: a cross-sectional survey. BMJ Open 2015;5:e006719.

51 Mannion R, Smith J, Culture H. Hospital culture and clinical performance: where next? BMJ Qual Saf 2018;27:179-81.

52 Sands KE. Patient-Centred care: confessions of a pragmatist. BMJ Qual Saf 2016;25:909-10.

53 Garon-Sayegh P. Analysis of medical malpractice claims to improve quality of care: cautionary remarks. J Eval Clin Pract 2019;25:744-50. 